\title{
A 16 pixel yaw sensor for velocity estimation
}

\author{
Sreeja Rajesh ${ }^{a, b, c}$, Derek Abbott ${ }^{a, c}$ and David C. O'Carroll ${ }^{b, c}$ \\ ${ }^{a}$ School of Electrical \& Electronic Engineering, The University of Adelaide, SA 5005, \\ Australia. \\ ${ }^{b}$ School of Molecular and Biomedical Science, The University of Adelaide, SA 5005, \\ Australia. \\ ${ }^{c}$ Centre for Biomedical Engineering, The University of Adelaide, SA 5005, Australia.
}

\begin{abstract}
The insect visual system, with its simplicity and efficiency has gained widespread attention and many biologically inspired models are being used for motion detection and velocity estimation tasks. One of the earliest and most efficient models among them is the Reichardt correlator model. In this paper, we have elaborated the basic Reichardt correlator to include spatial and temporal pre-filtering and additional non-linearites which are believed to be present in the fly visual system to develop a simple yaw sensor. We have used just 16 elaborated EMDs and it is seen that this sensor can detect rotational motion at angular velocities up to several thousand degrees per second. The modelling of these sensors make us realize that the VLSI implementation of such simple detectors can have varied applications for flight control in different fields.
\end{abstract}

\section{INTRODUCTION}

Despite their tiny brain, insects display excellent navigational skills and are able to detect motion and estimate velocity reliably. The efficiency of this system has stimulated the interest of engineers seeking inspiration for robots and other seeing machines. Several biologically inspired models of motion detection have been proposed.

Models of the visual systems of insects, with their relative simplicity and efficiency, have become the building blocks for improving the various techniques used in motion detection and velocity estimation. Of the various models of motion detection based on insect vision, the earliest and the most prominent model is the Reichardt correlator model which was developed by Hassenstein and Reichardt in $1956 .{ }^{1}$

The Reichardt correlation motion detector possess a highly parallel architecture. Each elementary motion detector (EMD) detects motion in a preferred direction by comparing a signal from one receptor with a delayed signal from an adjacent receptor. The comparison is performed using a nonlinear, multiplicative interaction between the two channels. Two EMDs tuned to opposite directions are combined to form a bidirectional motion detector.

Most of the spatiotemporal energy models - the dominant models for motion detection in vertebrates are mathematically equivalent to correlator models. ${ }^{2}$ Correlator models have been applied to explain motion detection in humans, birds and cats. ${ }^{3-5}$ Though insects and humans are capable of estimating image velocities, ${ }^{6,7}$ the basic correlator model does not function as a velocity estimator. It reliably indicates directional motion of sinusoidal gratings, but the response depends on contrast (brightness) and spatial frequency (shape) as well as velocity. ${ }^{8}$ Analysis and simulations suggest that the processes commonly found in visual systems, such as pre-filtering, response compression, integration, and adaptation, improve the reliability of velocity estimation and expand the range of velocities coded.$^{9-15}$ Hence we have elaborated the basic Reichardt model to mimic the properties of the insect visual system.

Further author information: (Send correspondence to Sreeja Rajesh)

Sreeja Rajesh, e-mail: srajesh@eleceng.adelaide.edu.au, Telephone: 8303-6296

David O'Carroll, e-mail: david.ocarroll@adelaide.edu.au

Derek Abbott, e-mail: dabbott@eleceng.adelaide.edu.au 


\section{MOTION DETECTION USING A REICHARDT CORRELATOR MODEL}

Figure 1 shows a simplified version of the correlator model. Receptors A and B are separated by an angular distance $\Delta \phi$. The signal $s_{1}(t)$ from $\mathrm{A}$ is temporally delayed by the low-pass filter $\mathrm{D}$ before multiplication by the signal $s_{2}(t)$ from B. This multiplication produces a positive output in response to rightward image motion. To achieve similar sensitivity to leftward motion and to cancel excitation by stationary flickering stimuli, a parallel delay-and-multiply operation takes place on the opposite arm. The outputs of the two multiplications are subtracted to give a single time-dependent correlator output $R$.

Although the correlator is nonlinear, its response to sinusoidal stimuli is of interest. If the input is a sinusoidal grating that contains only a single frequency component, the oscillations of the two subunits cancel and the correlator produces a constant output. For any linear delay filter, the output level depends separably on spatial and temporal frequency. ${ }^{8}$ If the delay filter $\mathrm{D}$ is first order low pass with time constant $\tau$, as in most modeling studies, a sinusoid of amplitude $C$ and spatial frequency $f_{s}$ traveling to the right at velocity $v$ produces an output

$$
R(t)=\frac{C^{2} f_{t}}{2 \pi \tau f_{t}^{2}+1 /(2 \pi \tau)^{2}} \sin \left(2 \pi f_{s} \Delta \phi\right)
$$

where $f_{t}=f_{s} v$ is the temporal frequency of the input signal. ${ }^{8}$ At a given spatial frequency, the magnitude of correlator output increases with temporal frequency up to an optimum $f_{t, \text { opt }}=1 /(2 \pi \tau)$ and then decreases monotonically as the velocity continues to increase. The output also varies with the square of $C$, which specifies grating brightness or, in the presence of preprocessing stages, grating contrast. A physical luminance grating must have positive mean luminance, so it will contain a dc component as well as an oscillatory component. In this case, the output will oscillate about the level given by Eq. (1).

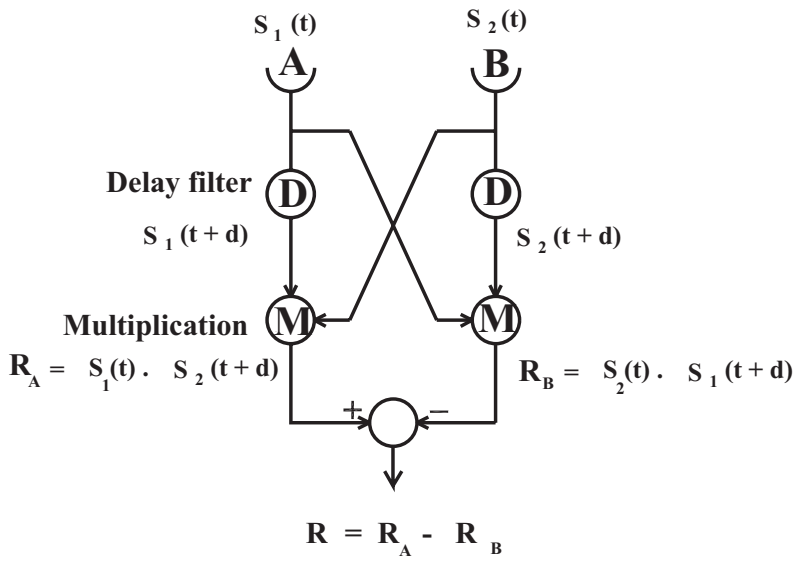

Figure 1. The Reichardt correlator has two receptors $A$ and $B$ that take two input signals $s_{1}$ and $s_{2}$ with a fixed angular separation $\Delta \phi$. Each of these time dependent inputs passes through a linear delay filter $(D)$ before being multiplied by the other, undelayed signal. The results of the two correlations thus obtained $R_{A}$ and $R_{B}$ are subtracted to produce a single output $R$. An object moving to the right will produce a positive output; an object moving to the left will produce a negative output. 


\subsection{Natural images used as stimuli}

Natural images are not arbitrary. Certain image statistics are highly predictable in the natural world ${ }^{16,17}$ and the biological visual system is optimized to take advantage of these statistics. ${ }^{10}$ In our experiments, natural images photographed from favored hovering positions of the hoverfly are used. Then the edges of the images are wrapped to form a panoramic image, which is given as a stimulus to our fly EMD model. A panorama is formed by 'warping' 12 image 'tiles' at $30^{\circ}$ intervals to remove lens distribution and then wrapping its ends together. This was done using Apple Quicktime VR Authoring Studio. The natural images used here in shown in Figure 2.

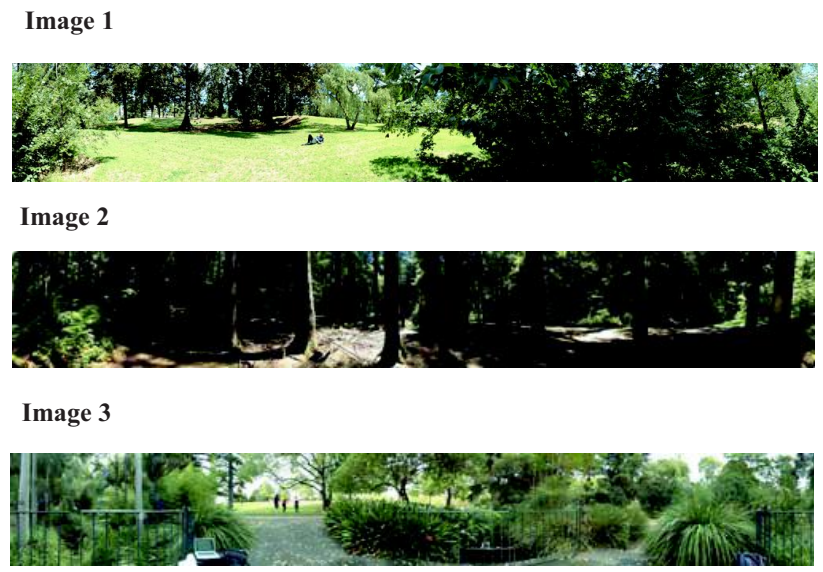

Fig. 2. The panoramic natural image given as stimulus to the EMD model. A panorama of the image is formed by 'warping' 12 image tiles at $30^{\circ}$ intervals to remove lens distortions and then by wrapping its ends together using Apple Quicktime VR software on a Macintosh computer.

\section{ELABORATED REICHARDT CORRELATOR}

Although the simple correlator model is able to detect motion very well, it is not a good velocity estimator, because its response depends on spatial structure of the image and contrast. Hence in an attempt to make its response independent of contrast and spatial frequency, we have elaborated the basic EMD model. Previous work shows that addition of various spatial and temporal pre-filtering, integration and adaptation mechanisms improve the performance of the correlator model ${ }^{17}$. The spatial low pass filtering is implemented using a Gaussian filter of half width $21.8^{\circ}$ and a temporal bandpass filter is implemented using a log-normal difference filter. $^{18}$

Spatial pre-filtering is implemented by two-dimensional convolution of the image with a Gaussian kernel of half width of $21.8^{\circ}$, which approximates ten times the acceptance function of typical fly photoreceptor and thus will be tuned to speeds approximately ten times the usual speed. ${ }^{19}$ Only the luminance (gray scale) information is taken from the image using the green channel, since photoreceptors are green sensitive. The distance between two ommatidia in an insect eye is between 1 and 1.5 degrees. Since the insect is looking at an image of 360 degrees, if we consider the inter-ommatidial angle as 22.5 degrees, there will be a total of 16 ommatidia looking at the image. So there will be an array of typically 16 EMDs working together to detect motion as shown in Figure 3.

The image is then temporally filtered with a log-normal difference filter to mimic the response of the lamina monopolar cells. ${ }^{18,20,21}$ The temporally pre-filtered image is then converted to a space-time matrix as shown in Figure 4 based on the desired velocity. Three rows of the EMDs are computed in the vertical dimension also with an inter-ommatidial angle of 22.5 degrees. 


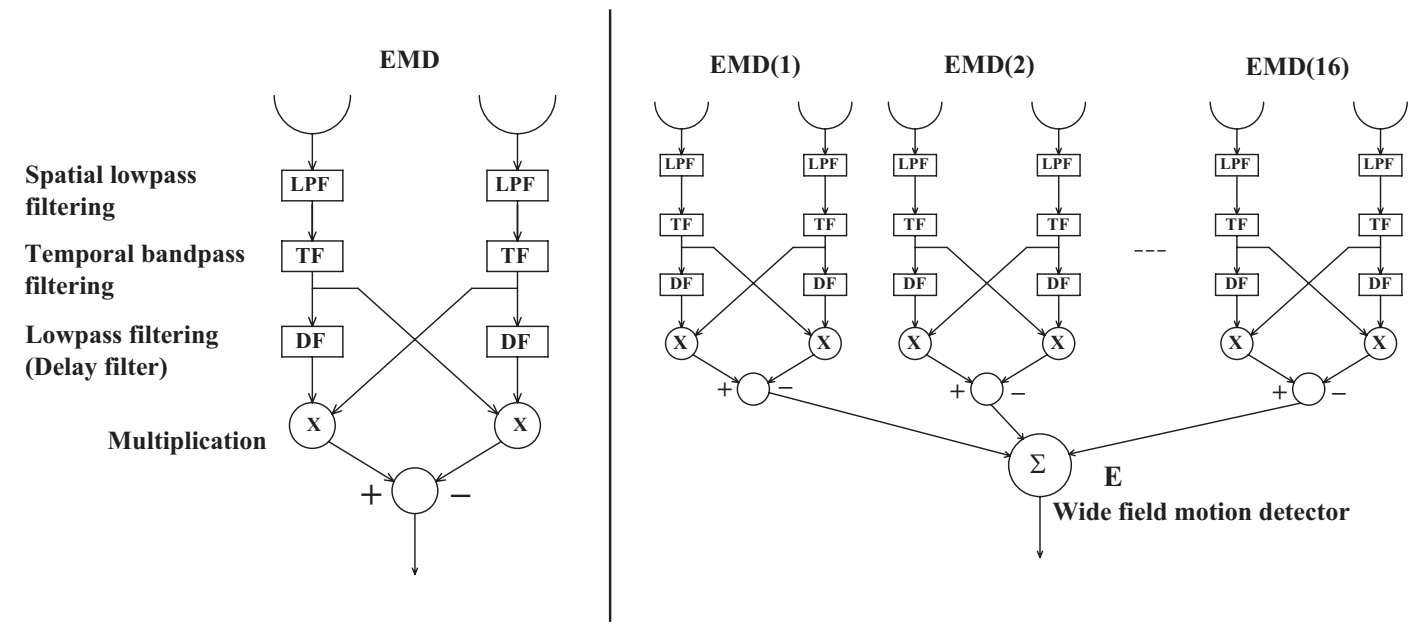

Figure 3. The elaborated EMD array. On the left, we have one elementary motion detector elaborated to include spatial low pass filtering (LPF) using a Gaussian filter of half width $21.8^{\circ}$ and a temporal band pass filter (TF) using a difference of log-normal filter is shown. This then passed through the delay and compare mechanism of the Reichardt detector using the the delay filter (DF) which is a low pass filter. On the right we have our EMD array model, where an array of 16 elaborated EMDs are used to detect motion. The input stimulus given is a panoramic image photographed from a natural environment favored by insects, and considering the inter ommatidial angle as $22.5^{\circ}$ there will be typically 16 ommatidia (EMDs) working together to detect motion. The output of these EMDs is pooled to get an average output (E) to enable wide field motion detection.

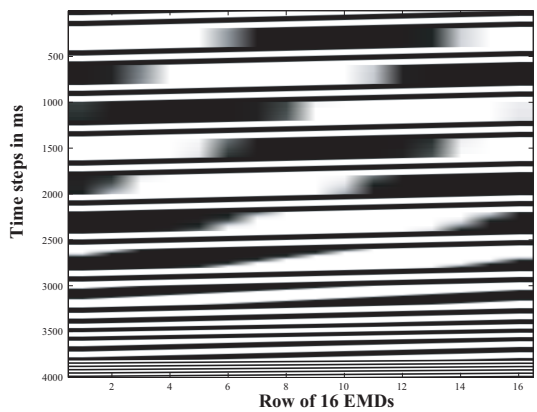

Figure 4. The panoramic image is rotated at a given velocity and is sampled on to an array of 16 EMDs separated by an inter-ommatidial angle of 22.5 degrees. The sample rate used here is 500 samples per second and the duration of the simulation is 8 seconds. The figure shows the image of the space time distribution or the spatio-temporal structure of a simulated single row of the image animated at a constant velocity of 1500 degrees/sec and sampled onto an array of 16 EMDs. Here the image used is image 1 (shown in Figure 2).

Then this spatio-temporally pre-filtered image is given to the EMD array, which correlates the inputs to give an array of outputs as is believed to take place in the insect eye. ${ }^{13,22}$ Then the EMD array model copies the lobula by averaging the outputs to produce an average EMD response.

\section{MOTION ADAPTATION IN THE FLY VISUAL SYSTEM}

When you observe a fly hovering near a flower, you will be struck by the ability of its visual system to estimate self-motion in order to achieve stabilization during hovering, which shows its sensitivity to low velocities. Similarly when you watch these insects engaging in high speed aerial pursuits while chasing mates, it is clear that their visual system adapts easily to higher velocities as well. These activities of insects demand a 
visual system with a large dynamic range. Although physiological recordings demonstrate that insect motion detectors have such high sensitivity to contrast that they are able to respond over a large range of velocities, this inherently high sensitivity to motion makes them prone to saturation. ${ }^{15,23}$

Several electrophysiological investigations into the dynamic response properties of fly motion detectors indicate the presence of motion adaptation.

Barlow and Hill $(1963)^{24}$ were the first to show that responses of directional motion sensitive neurons decline gradually during prolonged motion stimulation. They suggested that this decline in response and subsequent reduction in background activity that occurred after the stimulus stopped, may be correlated with psychophysically measured motion after effects. Maddess and Laughlin (1985) ${ }^{14}$ reported that adaptation does not depend on velocity but rather on temporal frequency of the stimulus, and may alleviate saturation.

Moreover, de Ruyter van Steveninck $(1986)^{25}$ found from his studies that the value of the time constant depends on the magnitude of local velocity from which he concludes that the fly visual system uses estimates of local stimulus velocity to tune its filtering operations. Borst and Egalhaaf $(1987)^{26}$ disagree with him over the origin of the adapting signal, suggesting that the adaptational state of a motion detector is governed mainly by the temporal frequency of the signal in its input channels.

Clifford and Langley (1995) ${ }^{27}$ in their adaptive Reichardt model, proposed that the adapting signal originates from locally integrated responses of a one dimensional array of elementary motion detectors, and is fed back to adapt the time constants of their temporal filters, suggesting that adaptation to higher velocities is achieved by shortening the delay filter in the correlator model. Ibbotson $(1998)^{28}$ tested this adaptive Reichardt model by recording from neurons in the wallaby visual system, finding little change in the position of temporal frequency optimum before and after motion adaptation, despite showing changes to image step responses similar to those in fly neurons. Furthermore recent studies in the fly by Harris et al $(1999)^{15}$ show little change in the temporal and spatial tuning properties of fly motion sensitive cells following adaptation, indicating that motion adaptation does not significantly alter the inherent velocity optimum of the EMDs. Instead this work reveals that motion adaptation induces a profound decrease in contrast sensitivity of fly motion sensitive cells via two proposed mechanisms, a local direction sensitive after potential and a local direction insensitive contrast control. We propose that this gain reduction may serve to reduce sensitivity to image contrast, as well as reducing tendency of the motion detectors to saturate.

\section{CONTRAST GAIN REDUCTION - FEEDBACK ADAPTIVE EMD MODEL}

In our elaborated Reichardt correlator array model, in order to remove the dependancy of the response to changes in contrast and spatial frequency and to get an accurate estimate of velocity, contrast gain reduction is implemented by a feedback adaptive process as in Fig. 5. The gain of the EMD inputs is reduced by a signal derived from the rectified and low pass filtered outputs of a local EMD pool with different local preferred direction, fed back to control the gain of the EMD inputs. The model was inspired by the observation of contrast dependent gain reduction in the responses of HS neurons following motion stimulation.? The model captures several aspects of the adaptive phenomena observed in the biological system. In particular, the adaptation is strongest when the local motion detector output is the largest, conferring a robustness in adaptation to motion signals as opposed to static flicker or noise applied to the inputs. This matches data obtained from the electrophysiological experiments, which show that motion stimuli are much more effective at recruiting adaptive gain than other stimuli. Secondly, the adaptive mechanisms remain independent of the direction of local motion, despite the selectivity for motion as the source of adaptation.

\section{ADDITION OF COMPRESSIVE NON-LINEARITY (SATURATION)}

Both simple and elaborated Reichardt correlators show an increase of response amplitude with stimulus contrast. The neural and behavioral responses of the fly display such a dependence only at very low contrasts. 


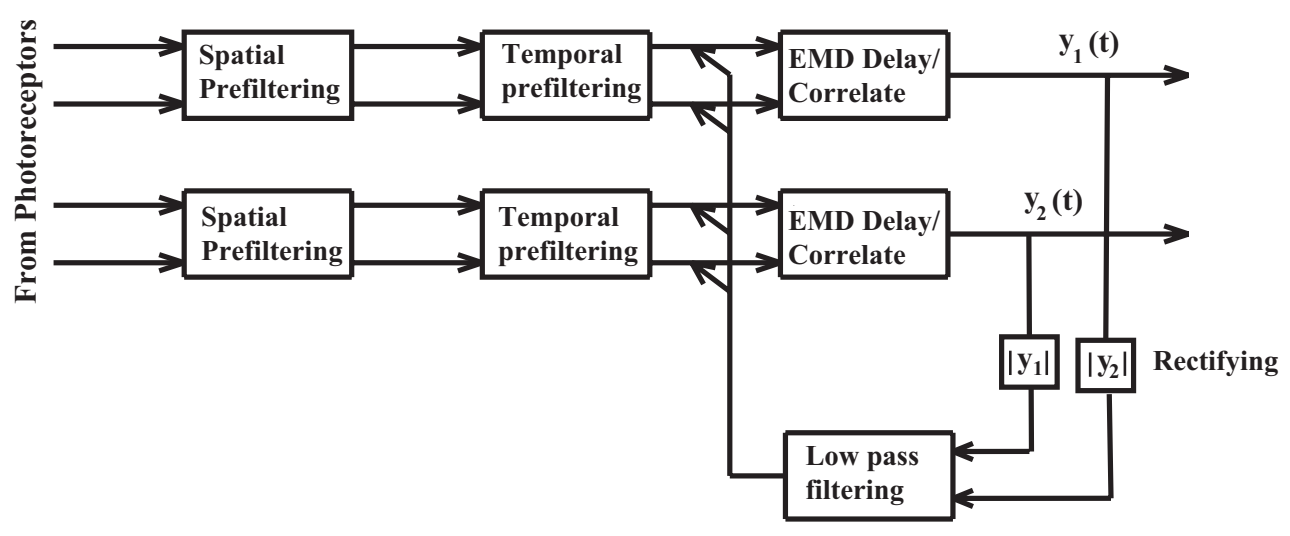

Fig. 5. Block diagram of a feed back adaptive EMD model. In order to reduce the dependency of the response to contrast we have implemented a further elaboration to this model that reduces contrast gain by a feedback adaptive mechanism. The output signals $y_{1}(t)$ and $y_{2}(t)$ are rectified, low pass filtered and fed back to control the gain of the EMD inputs. So depending upon the outputs of the EMd, the input to the Reichardt model is controlled. The model captures several aspects of the adaptive phenomena observed in the biological system. In particular, the adaptation is strongest when the local motion detector output is the largest, conferring a robustness in adaptation to motion signals as opposed to static flicker or noise applied to the inputs. This matches data obtained from the electrophysiological experiments, which show that motion stimuli are much more effective at recruiting adaptive gain than other stimuli. Secondly, the adaptive mechanisms remain independent of the direction of local motion, despite the selectivity for motion as the source of adaptation.

As contrast increases above a few percent, the response begins to level off due to a static, compressive nonlinearity which is termed as contrast saturation. ${ }^{29}$ This is due to limitations in the range of responses that can be signaled by physiological mechanisms.

\subsection{Saturation at the correlator input}

Saturation of the visual signal first occurs in the photoreceptors, which respond roughly to logarithm of luminance. ${ }^{10}$ Saturation reduces relative error partly by reducing contrast difference from one region of the image to another. It is seen in flies that this saturation occurs primarily after linear pre-filtering but before the multiplication operation indicating that contrast saturation must take place after elimination of the mean light intensity from the signal. 8,29 Saturation is modelled here by including a compressive non-linearity such as a hyperbolic tangent function of the form, $\rho(C)=\tanh (C)$.

\subsection{Saturation at the correlator arms}

It is also seen that saturation also takes place on both the delayed and undelayed arms of the correlator with saturation on the delayed arm following the delay filter. ${ }^{8,29}$ So based on this, we have implemented compressive nonlinearity before the multiplication operation on both correlator arms.

\subsection{Saturation at the output}

The outputs of the wide field neurons are also found to saturate due to shunting of the membrane potential. ${ }^{12}$ This is introduced as a compressive non-linearity following spatial integration. ${ }^{29}$ Such an effect will flatten the peaks of the velocity response curves effectively allowing neuron to use more of its dynamic range to signal low velocities. So based on this we have implemented saturation at the output stage of our model. 


\section{RESULTS}

The fully elaborated 16 EMD array model is then simulated using the natural image stimuli shown in Figure 2. To test the motion adapted responses, we increased velocity step-wise, with interleaved bursts of adapting motion (constant speed). The velocity is increased in steps with time as shown in Figure 6. The mean response of the EMD array model with and without output saturation is shown in Figure 7.

Figure 8 shows the mean correlator response of three rows of EMDs. The dotted line which shows the mean EMD output of the first or top row of 16 EMDs could be considered as the response of the HSN neuron of the fly tangential cell, the second row or the middle row shown by the dashed line could be the response of the HSE and the third row or the bottom row mean correlator response could be the response of the HSS neuron of the fly tangential cell. The mean response of each row is sensitive to the degree of contrast that is seen in the elevation of the natural scene that it is oriented towards. And finally Figure 9 shows the velocity response curve of EMD array for the three different images shown in Figure 2. It is seen that since image 1 and image 2 have high contrast features, their velocity response curves overlap. For Image 3, which has little in terms of high contrast features, the velocity response curve is consequently slightly lower as seen in Figure 9. The naturalistic three images which we have chosen differ in aggregated natural contrast (global contrast) approximately by a factor of 3 (Image 1 has a global contrast, $C n=0.65$, Image 2 has $C n=1$ and for Image $3, C n=0.38$ as calculated by Straw $\left.^{30}\right)$. Since we know that the response of a simple Reichardt correlator model varies quadratically with contrast, ${ }^{29}$ it is seen that the addition of the non-linear elaborations in our elaborated model decreases the contrast dependency of the correlator response considerably. And it is also seen that this small sensor can detect angular velocities up to several thousand degrees per second.

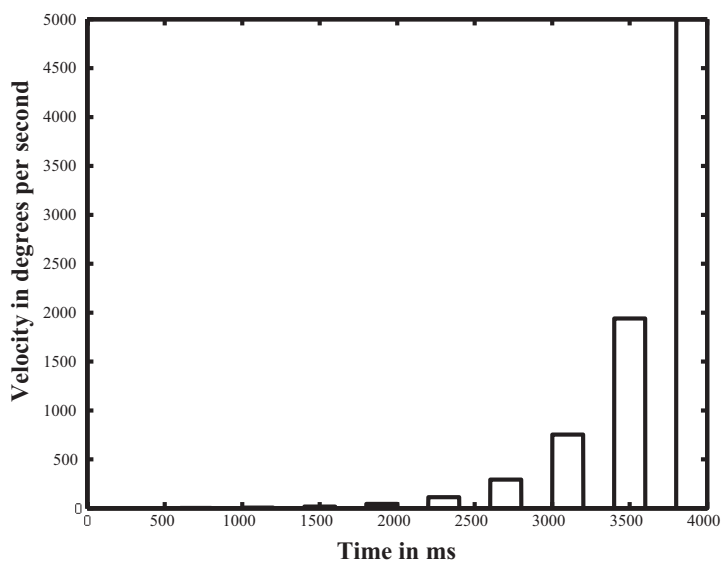

Fig. 6. To test motion adapted responses, we increased velocity step-wise, with interleaved bursts of adapting motion (constant speed). The velocity is increased in steps with time

\section{CONCLUSION}

In this paper, we have developed new variants of the Reichardt correlator model, elaborated to incorporate additional non-linearities that mimic known properties of the insect motion pathway, including logarithmic encoding of luminance, saturation and motion adaptation (adaptive gain-control). Here, we have modeled the response of a small circular detector array consisting of 16 elaborated EMDs configured in such a way as to provide a sensor tuned to the yaw component of optical flow. Although this small detector array has limited spatial resolution, this confers an advantage (at least using reasonably short time constants for the delay element) that it can provide monotonic responses to yaw stimuli at angular velocities up to several thousand degrees per second. The circular configuration of the detector array is capable of rejecting the phase-specific variation in response to complex patterns that typifies Reichardt-type motion detectors. Although a simple 


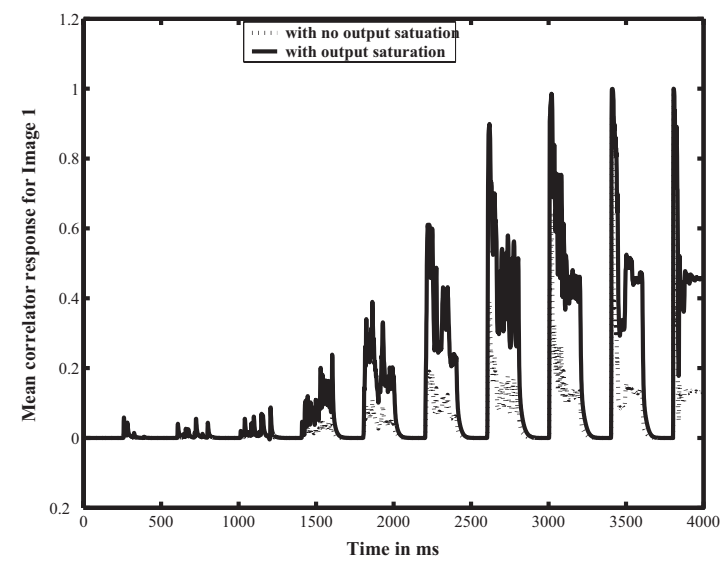

Fig. 7. This figure shows the total mean correlator response without implementing output saturation and with output saturation. Inclusion of output saturation will flatten the peaks of the velocity response curves, effectively allowing the neuron to use more of its dynamic range to code for low velocities

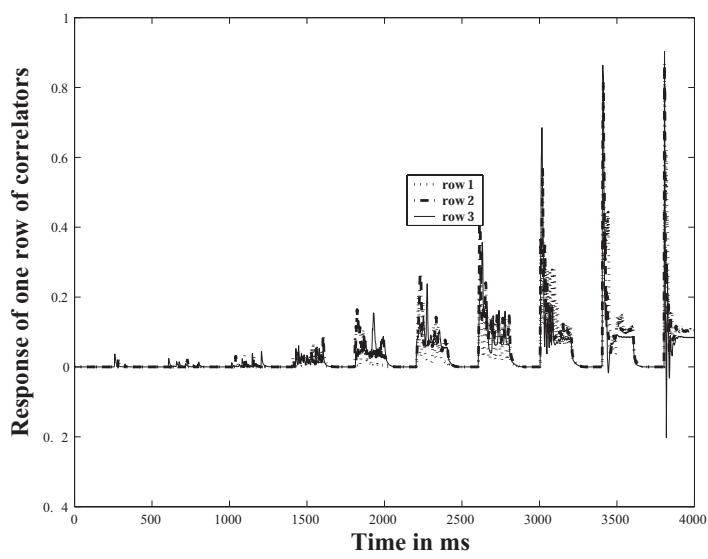

Fig. 8. This figure shows the mean correlator response of three rows of EMDs. The response of each row is seen to vary with the contrast found at that elevation. 


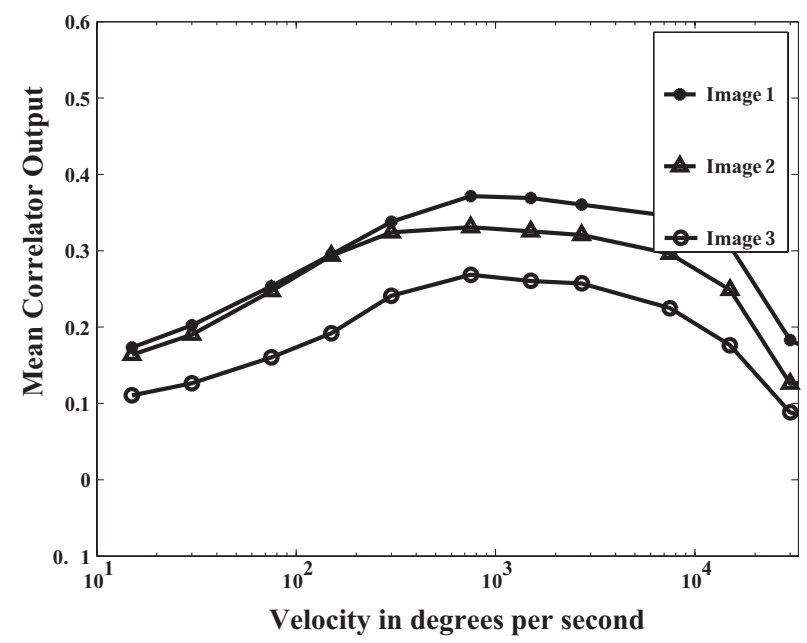

Fig. 9. This figure shows the velocity response curve of the 16 pixel sensor for three images shown in Figure 2 . The addition of the non-linearities decreases the contrast dependency of the sensor and this small detector array is capable of estimating angular velocities upto several thousand degrees per second.

1-dimensional (circular) array of Reichardt EMDs would still be sensitive to the degree of contrast that prevails in a scene at the elevation it is oriented towards, the non-linear elaborations to our modeled array largely negate such variation. Hence, our model demonstrates that it would be feasible to base a biomimetic motion detector for sensing rotational components of optical flow on a very small number of sampled pixels. A group of such sensors, implemented in analog VLSI would have applications for flight control in ultra-miniature unmanned aerial vehicles.

\section{Acknowledgments}

This project was supported by US Air force for Scientific Research /AOARD (contract \# F62562-01-P-0158) and US Air force SBIR contract (contract \# F 08630-01-C-0050).

\section{REFERENCES}

1. B. Hassenstein and W. Reichardt, "Structure of a mechanism of perception of optical movement," Proceedings of the 1st International Conference on Cybernetics, pp. 797-801, 1956.

2. E. H. Adelson and J. Bergen, "Spatiotemporal energy models for the perception of motion," Journal of the Optical Society of America A 2, pp. 284-299, 1985.

3. J. P. van Santen and G. Sperling, "Elaborated Reichardt detectors," Journal of the Optical Society of America A 2, pp. 300-321, 1985.

4. F. Wolf-Oberhollenzer and K. Kirschfeld, "Motion sensitivity in the nucleus of the basal optic root of the pigeon," Journal of Neurophysiology 71, pp. 1559-1573, 1994.

5. R. C. Emerson, M. C. Citron, W. J. Vaughn, and S. A. Klein, "Nonlinear directionally selective subunits in complex cells of cat striate cortex," Journal of Neurophysiology 58, pp. 33-65, 1987.

6. M. V. Srinivasan, S. W. Zhang, M. Lehrer, and T. S. Collet, "Honeybee navigation en route to the goal: visual flight control and odometry," Journal of Experimental Biology 199, pp. 237-244, 1996.

7. S. P. McKee, G. H. Silverman, and K. Nakayama, "Precise velocity discrimination despite random variation in temporal frequency and contrast," Vision Research 26, pp. 609-619, 1986.

8. M. Egelhaaf, A. Borst, and W. Reichardt, "Computational structure of a biological motion detection system as revealed by local detector analysis in the fly's nervous system," Journal of the Optical Society of America A 6, pp. 1070-1087, 1989. 
9. R. O. Dror, D. C. O'Carroll, and S. B. Laughlin, "Accuracy of velocity estimation by Reichardt correlators," Journal of the Optical Society of America A 18, pp. 241-252, February 2001.

10. S. B. Laughlin, "Matching coding, circuits, cells and molecules to signals: general principles of retinal design in the fly's eye," Progress in Retinal Research 13, pp. 165-195, 1994.

11. M. Egelhaaf and A. Borst, "Transient and steady state response properties of movement detectors," Journal of the Optical Society of America A 6, pp. 116-127, 1989.

12. S. Single and A. Borst, "Dendritic integration and its role in computing image velocity," Science 281, pp. 1848-1850, 1998.

13. K. Hausen and M. Egelhaaf, Neural mechanisms of visual course control in insects, Facets in vision, edited by R. Hardie and D. Stavenga, Springer-Verlag, Berlin, 1989.

14. T. Maddess and S. B. Laughlin, "Adaptation of the motion sensitive neuron h1 is generated locally and governed by contrast frequency," Proceedings of the Royal Society of London B 225, pp. 251-275, 1985.

15. R. A. Harris, D. C. O'Carroll, and S. B. Laughlin, "Adaptation and the temporal delay filter of fly motion detectors," Vision Research 39, pp. 2603-2613, 1999.

16. D. L. Ruderman, "The statistics of natural images," Network: Computation in Neural System 5, pp. 517548, 1994.

17. R. O. Dror, D. C. O'Carroll, and S. B. Laughlin, "The role of natural image statistics in biological motion estimation," Proceedings of the IEEE International Workshop on Biologically Motivated Computer Vision, Seoul, Korea 1811, pp. 492-501, 2000.

18. A. C. James, White-Noise Studies in the Fly Lamina. PhD thesis, Australian National University, 1990.

19. R. C. Hardie, "Functional organisation of the fly retina," Progress in sensory physiology 5, pp. 1-80, 1985.

20. R. Payne and J. Howard, "Response of an insect photoreceptor: a simple log-normal model," Nature 290, pp. 415-416, 1981.

21. J. H. Howard, A. Dubs, and R. Payne, "The dynamics of phototransduction in insects," Journal of Comparative Physiology 154, pp. 707-718, 1984.

22. J. K. Douglass and N. J. Strausfeld, "Visual motion detection circuits in flies: peripheral motion computation by identified small-field retinotopic neurons," Journal of Neuroscience 15, pp. 5596-5611, 1995.

23. R. A. Harris, D. C. O'Carroll, and S. B. Laughlin, "Contrast gain reduction in fly motion adaptation," Neuron 28, pp. 595-606, November 2000.

24. H. B. Barlow and R. M. Hill, "Evidence for a physiological explanation of the waterfall phenomenon and figural after-effects," Nature 200, pp. 1345-1347, 1963.

25. R. R. de Ruyter van Steveninck, W. Zaagman, and H. A. K. Mastebroek, "Adaptation of transient responses of a movement sensitive neuron in the visual system of blowfly Caliphora erythrocephala," Biological Cybernetics 54, pp. 223-236, 1986.

26. A. Borst and M. Egelhaaf, "Temporal modulation of luminance adapts time constant of fly movement detectors," Biological Cybernetics 56, pp. 209-215, 1987.

27. C. W. G. Clifford and K. Langley, "A model of temporal adaptation in fly motion vision," Vision Research 36(16), pp. 2595-2608, 1995.

28. M. R. Ibbotson, C. W. G. Clifford, and R. F. Mark, "Adaptation to visual motion in directional neurons of the nucleus of the optic tract," Journal of Neurophysiology 79, pp. 1481-1493, 1998.

29. R. O. Dror, "Accuracy of visual velocity estimation by Reichardt correlators," Master's thesis, University of Cambridge, Cambridge, UK, 1998.

30. A. Straw, Neural responses to moving natural scenes. PhD thesis, University of Adelaide, Australia, 2004. 